\title{
States and Dynamics of Infinitely Extended Physical Systems
}

\author{
G. L. Sewell \\ Department of Physics, Queen Mary College, London, U.K.
}

Received March 12, 1973

\begin{abstract}
A scheme is presented for the description of the states and dynamics of infinitely extended systems. In this scheme, the physical states of a system are taken to comprise the maximal folium of its locally normal states which can support a one-parameter group of affine transformations, that corresponds to a certain infinite volume limit of the time-translational group for the states of a finite system of particles of the same species. The resultant one-parameter group of transformations of the physical states of the infinitely extended system is then taken to correspond to its time-translations. An explicit construction is given which serves to identify the physical states and dynamics of the system in terms of its interactions. The present scheme generalises that of Dubin and the author beyond the islands of Gibbs states.
\end{abstract}

\section{Introduction}

It is well-known that the generalisation of quantum or classical dynamical laws to infinitely extended physical systems presents certain problems (cf. $[1,2,3]$ ). A first approach to a dynamical theory for such systems was made within the $C^{*}$-algebraic framework by Haag, Hugenholtz, and Winnink (HHW) [4]. The scheme of HHW was based on a postulate concerning the existence of a certain "infinite volume limit," which led to a description of time-translations of an infinite system in terms of a one-parameter group of automorphisms of the $C^{*}$-algebra of its observables. However, although the basic postulate of HHW is satisfied by lattice systems with suitably tempered interactions [5], it is not generally valid for non-relativistic continuous systems [1] or for lattice systems with sufficiently long range interactions [2].

A subsequent approach to the dynamical problem by Dubin and the present author (DS) [1], based on weaker assumptions than those of $\mathrm{HHW}$, led to a description of time-translations in the "island" of a Gibbs state in terms of a one-parameter group of automorphisms of the weak closure of the associated GNS representation of the algebra of observables. However, although the DS scheme is applicable to a wider class of systems than that of HHW, it has the disadvantage of being strictly limited to the islands of Gibbs states. This limitation is serious 
for the following two reasons. Firstly, it rules out the possibility of formulating the dynamics of a system in other states, e.g. those which are appropriately "far" from equilibrium. Secondly, it precludes the possibility of deriving equilibrium conditions for an infinite system in terms of some suitable dynamical principle: this is a drawback because the Gibbs states are merely defined as thermodynamical limits of finitevolume canonical (or grand canonical) states, whose identification with equilibrium states has not, as yet, been established on a fundamental basis.

The object of the present article is to construct a dynamical theory for infinitely extended systems, without resorting to assumptions concerning equilibrium states (e.g., such as KMS conditions). The theory is constructed within the $C^{*}$-algebraic formalism and is based on the postulate that the physical states of a system constitute the maximal folium (defined in Ref. [6]) of its locally normal states which can support a one-parameter group of affine transformations that corresponds to a certain infinite volume limiting form of the time-translational group for a finite system of particles of the same species. The resultant one-parameter group of affine transformations of the physical states is taken to correspond to the time-translations ${ }^{1}$ of the system. On the basis of the above-described postulate, we are able to formulate an explicit construction of the set of physical states of the system and of its timetranslation group. It will be seen that this construction generalises the DS scheme beyond the islands of the Gibbs states.

The material of this article will be presented as follows. In Section 2, we introduce the concept of a dynamical folium, i.e. of a folium equipped with a one-parameter group of affine transformations, which do not necessarily correspond to time-translations in a physical sense. We also define certain classes of dynamical folia. In Section 3, we introduce our basic postulate that the physical folia are those locally normal dynamical folia whose dynamical groups correspond to appropriately defined infinite volume limits of corresponding groups for finite systems (cf. Definition 3.1). This postulate enables us to obtain an explicit construction for the physical folia and their time-translation groups (Proposition 3.1) in terms of the interactions in the system. Our main results are given by Proposition 3.1 and the subsequent discussion in Section 3. In Appendix 1, we present the proof of Proposition 3.1. In Appendix 2, we apply our scheme to the formulation of time-translations in certain folia of states of an ideal bose gas.

\footnotetext{
${ }^{1}$ A quite different approach to the theory of time-translations has been made by Lanford [3], who has formulated the dynamics of a certain class of states of an infinitely extended one-dimensional classical system on the basis of the Newtonian equations of motion for its constituent particles.
} 


\section{Observables and Dynamical Folia}

We start by recalling that the set of observables of an infinitely extended system may be constructed as follows (cf. [4]). Let $\Gamma$ be the one-particle space (e.g., $R^{v}$ or $Z^{v}$ ) of the system, and let $L$ be the set $\{\Lambda\}$ of bounded measurable open subsets of $\Gamma$. By a standard procedure, one may construct a Fock-Hilbert space $\mathscr{H}$ over $\Gamma$, corresponding to the species of the particles in the system, assigning to each $\Lambda$ in $L$ a subspace $\mathscr{H}_{A}$ of $\mathscr{H}$ and a $C^{*}$-algebra $\mathscr{A}_{A}$ in $\mathscr{B}\left(\mathscr{H}_{A}\right)$ such that $\mathscr{H}_{A}$ and $\mathscr{A}_{A}$ are isotonic with respect to $\Lambda$ : here $\mathscr{A}_{A}$ is chosen so that its self-adjoint elements correspond to the bounded observables of an assembly of particles of the given species, confined to the region $\Lambda$. We define $\mathscr{A}_{L}$ to be $\bigcup_{A \in L} \mathscr{A}_{\Lambda}$ and $\mathscr{A}$ to be the norm-completion of $\mathscr{A}_{L}$. The self-adjoint elements of $\mathscr{A}_{L}$ (or of $\mathscr{A}$ ) are taken to comprise the set of bounded ${ }^{2}$ observables of the system.

Let $\mathscr{A}^{*}$ (resp. $\mathscr{A}_{+}^{*}$ ) be the set of all (resp. positive) continuous linear functionals on $\mathscr{A}$, and let $\mathscr{L}$ be the set of elements of $\mathscr{A}_{+}^{*}$ whose restrictions to the subalgebras $\left\{\mathscr{A}_{\Lambda} \mid \Lambda \in L\right\}$ are all normal; i.e., in a usual terminology, $\mathscr{L}$ is the set of locally normal elements of $\mathscr{A}_{+}^{*}$. We define $\alpha$ to be the map from $\mathscr{A}$ into the affine transformations of $\mathscr{A}_{+}^{*}$ given by the formula

$$
\langle\alpha(A) \phi ;(\cdot)\rangle=\left\langle\phi ; A^{*}(\cdot) A\right\rangle, \quad \forall \phi \in \mathscr{A}_{+}^{*}, \quad A \in \mathscr{A} .
$$

Then, following Haag, Kadison, and Kastler [6], we term a subset $\mathscr{I}$ of $\mathscr{A}_{+}^{*}$ a folium if $\mathscr{I}$ is norm-closed, stable under $\alpha(\mathscr{A})$ and closed under convex combinations. Thus, for example, $\mathscr{L}$ is a folium. If $K$ is some subset of $\mathscr{A}_{+}^{*}$, we define the folium generated by $K$ to be the smallest folium containing $K$. If $\mathscr{I}_{1}, \mathscr{I}_{2}$ are folia in $\mathscr{A}_{+}^{*}$, such that $\mathscr{I}_{1} \subset \mathscr{I}_{2}$, we say that $\mathscr{I}_{1}$ is a subfolium of $\mathscr{I}_{2}$. In particular, if $\mathscr{I}$ is a subfolium of $\mathscr{L}$, we say that $\mathscr{I}$ is a locally normal folium.

Let $\mathscr{I}$ be a folium in $\mathscr{A}_{+}^{*}$. We define $[\mathscr{I}]$ to be the norm-closed linear span of $\mathscr{I}$, and $[\mathscr{I}]^{*}$ to be the dual space of $[\mathscr{I}]$. We define the map $A \rightarrow A_{\mathscr{I}}$ of $\mathscr{A}$ into $[\mathscr{I}]^{*}$ by the formula

$$
\left\langle A_{\mathscr{I}} ; \phi\right\rangle=\langle\phi ; A\rangle, \quad \forall \phi \in \mathscr{I} ;
$$

and we define $\mathscr{A}_{\mathscr{I}}$ to be the image of $\mathscr{A}$ under this map. Let $\pi_{\mathscr{I}}$ be the direct sum of the GNS representations of $\mathscr{A}$ induced by the elements of $\mathscr{I}$. Then $\pi_{\mathscr{I}}(\mathscr{A})$ is a $C^{*}$-algebra in a certain Hilbert space $\mathscr{H}_{\mathscr{I}}$ and (cf. [6]) $\mathscr{A}_{\mathscr{I}}$ (resp. $\left.[\mathscr{I}]^{*}\right)$ is canonically isometric with $\pi_{\mathscr{I}}(\mathscr{A})$ (resp. $\left.\pi_{\mathscr{I}}(\mathscr{A})^{\prime \prime}\right)$. Thus, $[\mathscr{I}]^{*}$ is a $W^{*}$-algebra and $\mathscr{A}_{\mathscr{I}}$ is $\sigma\left([\mathscr{I}]^{*},[\mathscr{I}]\right)$ dense in $[\mathscr{I}]^{*}$.

\footnotetext{
${ }^{2}$ One may extend the above description by defining the set of all observables of the system, including the unbounded ones, to be $\bigcup_{A \in L} \mathscr{Q}_{\Lambda}$, where $\mathscr{Q}_{\Lambda}$ is the set of self-adjoint operators affiliated to $\mathscr{A}_{\Lambda}$ in $\mathscr{H}[7]$.
} 
In the particular case where $\mathscr{I}$ is generated by a single element $\omega$ of $\mathscr{A}_{+}^{*}, \mathscr{A}_{\mathscr{A}}$ corresponds to the GNS representation $\pi_{\omega}(\mathscr{A})$ induced by $\omega$, and $[\mathscr{I}]^{*}$ corresponds to $\pi_{\omega}(\mathscr{A})^{\prime \prime}$.

We define a dynamical folium to be a pair $\left(\mathscr{I}, \tau_{\mathscr{I}}\right)$ where $\mathscr{I}$ is a folium and $\tau_{\mathscr{f}}$ a homomorphism of the real line $R$ into the affine norm-preserving transformations of $\mathscr{I}$. We refer to the image $\tau_{\mathscr{I}}(R)$ of $R$ under such a homomorphism as a dynamical group for $\mathscr{I}$. We say that a dynamical folium $\left(\mathscr{I}, \tau_{\mathscr{I}}\right)$ is locally normal if $\mathscr{I} \subset \mathscr{L}$. Let $\left(\mathscr{I}, \tau_{\mathscr{I}}\right)$ and $\left(\mathscr{I}^{\prime}, \tau_{\mathscr{I}^{\prime}}\right)$ be two dynamical folia. We say that these dynamical folia are mutually compatible if either $\mathscr{I}$ and $\mathscr{I}^{\prime}$ are mutually disjoint or if $\tau_{\mathscr{I}}(t)$ and $\tau_{\mathscr{I}^{\prime}}(t)$ coincide on $\mathscr{I} \cap \mathscr{I}^{\prime}$, for all $t \in R$. We term $\left(\mathscr{I}, \tau_{\mathscr{I}}\right)$ a dynamical subfolium of $\left(\mathscr{I}^{\prime}, \tau_{\mathscr{I}^{\prime}}\right)$ if $\mathscr{I} \subset \mathscr{I}^{\prime}$ and $\tau_{\mathscr{I}}(t)=\tau_{\mathscr{I}}(t)_{\mathscr{I}}, \forall t \in R$.

Let $\left(\mathscr{I}, \tau_{\mathscr{I}}\right)$ be a dynamical folium. We define the one-parameter group $\left\{\tau_{\mathscr{I}}^{*}(t) \mid t \in R\right\}$ of transformations of $[\mathscr{I}]^{*}$, dual to $\tau_{\mathscr{I}}(R)$, by the formula

$$
\left\langle\tau_{\mathscr{I}}^{*}(t) F ; \phi\right\rangle=\left\langle F ; \tau_{\mathscr{I}}(t) \phi\right\rangle, \quad \forall t \in R, \phi \in \mathscr{I}, \quad F \in[\mathscr{I}]^{*} .
$$

It follows from this definition and a theorem due to Kadison [8; Corollary 4.7] that $\tau_{\mathscr{I}}^{*}(R)$ is a group of $C^{*}$-automorphisms of the $C^{*}$-algebra $[\mathscr{I}]^{*}$, i.e. that

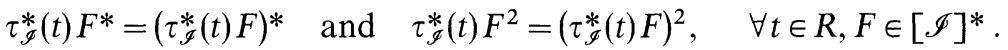

On the other hand, the transformations $\tau_{\mathscr{I}}^{*}(R)$ are not, in general, automorphisms of $[\mathscr{I}]^{*}$. In those cases where $\tau_{\mathscr{I}}^{*}(R) \subset$ Aut $[\mathscr{I}]^{*}$, we shall term the dynamical folium $\left(\mathscr{I}, \tau_{\mathscr{I}}\right)$ canonical. Thus, by Ref. [8; Theorem 3.4], $\left(\mathscr{I}, \tau_{\mathscr{I}}\right)$ is canonical in cases where $\mathscr{I}$ is generated by a mutually disjoint set of factor states and where the map $t(\in R) \rightarrow\left\langle F ; \tau_{\mathscr{I}}(t) \phi\right\rangle$ is continuous, for all $\phi \in \mathscr{I}, F \in[\mathscr{I}]^{*}$.

\section{Physical Folia}

We now seek to characterise those dynamical folia which correspond to physical states and time-translations of the system. For this purpose, we introduce a construction leading to a definition of a physical dynamical group for the infinitely extended system as a limiting form of a corresponding dynamical group for a finite system of particles of the same species, endowed with given forces.

Thus, for each $\Lambda \in L$, we define $H_{A}$ to be the self-adjoint operator in $\mathscr{H}_{A}$ corresponding to the Hamiltonian for an assembly of particles of the given species and with prescribed interactions, confined to the region $\Lambda$ and subjected to some definite $\Lambda$-independent boundary conditions, e.g., those corresponding to rigid walls. It is assumed that, for each $\Lambda \in L$ and $t \in R$, the transformation $A\left(\in \mathscr{A}_{\Lambda}^{\prime \prime}\right) \rightarrow e^{i H_{\Lambda} t} A e^{-i H_{\Lambda} t}$ 
corresponds to an automorphism of $\mathscr{A}_{\Lambda}^{\prime \prime}$. Thus, for each $\Lambda \in L$, we may define an homomorphism $\tau_{A}^{*}$ of $R$ into Aut $\mathscr{A}_{\Lambda}^{\prime \prime}$ by the formula

$$
\tau_{\Lambda}^{*}(t) A=e^{i H_{\Lambda} t} A e^{-i H_{A} t}, \quad \forall t \in R, \quad A \in \mathscr{A}_{\Lambda}^{\prime \prime} .
$$

Let $\phi \in \mathscr{L}$. Then $\phi_{\mid \Lambda}$ is normal for all $\Lambda \in L$ and consequently $\phi_{\mid \Lambda}$ may be extended by continuity from $\mathscr{A}_{\Lambda}$ to $\mathscr{A}_{\Lambda}^{\prime \prime}$. This extension thus serves to define $\left\langle\phi ; \tau_{A}^{*}(t) A\right\rangle$ for all $t \in R, A \in \mathscr{A}_{\Lambda}$. Therefore, since $\mathscr{A}_{A}$ is isotonic with respect to $\Lambda$, it follows that for each $A \in \mathscr{A}_{L}, \phi \in \mathscr{L}$ and $t \in R$, the quantity $\left\langle\phi ; \tau_{\Lambda}^{*}(t) A\right\rangle$ is well-defined for sufficiently large $\Lambda$.

Definition 3.1. Let $\left(\mathscr{I}, \tau_{\mathscr{J}}\right)$ be a dynamical folium in $\mathscr{A}_{+}^{*}$. We say that $\left(\mathscr{I}, \tau_{\mathscr{I}}\right)$ is a physical folium if it is locally normal and if

$$
\left\langle\tau_{\mathscr{I}}(t) \phi ; A\right\rangle=\lim _{A \rightarrow \infty}\left\langle\phi ; \tau_{\Lambda}^{*}(t) A\right\rangle, \quad \forall t \in R, \quad \phi \in \mathscr{I}, \quad A \in \mathscr{A}_{L},
$$

where $\lim _{\Lambda \rightarrow \infty}$ refers to the limit over an increasing absorbing sequence of regions $\Lambda(\in L)$, whose forms may be subjected to some prescribed restriction (e.g., that of Fisher [9]).

Note. Eq. (3.2) signifies precisely that, for each $\Lambda_{0} \in L$,

$$
\left(\tau_{\mathscr{I}}(t) \phi\right)_{\mid \Lambda_{0}} \equiv w^{*} \lim _{\Lambda \rightarrow \infty}\left(\tau_{\Lambda}(t) \phi_{\mid \Lambda}\right)_{\mid \Lambda_{0}},
$$

where $\tau_{\Lambda}(R)$ is the group of transformations of the normal linear functionals on $\mathscr{A}_{\Lambda}^{\prime \prime}$, dual to $\tau_{\Lambda}^{*}(\mathrm{R})$.

In order to prescribe a constructive method of specifying the physical folia, we introduce the following definition.

Definition 3.2. (i) We define $\mathscr{S}_{0}$ to be the set of elements $\phi$ in $\mathscr{L}$ such that $\lim _{\Lambda \rightarrow \infty}\left\langle\phi ; \tau_{A}^{*}(t) A\right\rangle$ exists for all $A \in \mathscr{A}_{L}, t \in R$, where $\lim _{\Lambda \rightarrow \infty}$ is taken in the same sense as in Definition 3.1. Thus, for each $t \in R$, we may define $\tau(t): \mathscr{S}_{0} \rightarrow \mathscr{A}_{+}^{*}$ by the formula

$$
\langle\tau(t) \phi ; A\rangle=\lim _{A \rightarrow \infty}\left\langle\phi ; \tau_{\Lambda}^{*}(t) A\right\rangle, \quad \forall \phi \in \mathscr{S}_{0}, \quad A \in \mathscr{A}_{L} .
$$

(ii) We define

$$
\begin{aligned}
\mathscr{S}_{1} & =\left\{\phi \mid \phi \in \mathscr{S}_{0} ; \tau(t) \phi \in \mathscr{S}_{0}, \forall t \in R ; \tau\left(t_{1}\right) \tau\left(t_{2}\right) \phi\right. \\
& \left.=\tau\left(t_{1}+t_{2}\right) \phi, \forall t_{1}, t_{2} \in R\right\} .
\end{aligned}
$$

(iii) We define $\mathscr{S}_{n}$, for integers $n>1$, by the following recursion formula:

$$
\mathscr{S}_{n}=\left\{\phi \mid \phi \in \mathscr{S}_{n-1} ; \alpha(A) \tau(t) \phi \in \mathscr{S}_{n-1}, \forall t \in R, A \in \mathscr{A}\right\} .
$$

(iv) We define $\mathscr{S}=\bigcap_{n=0}^{\infty} \mathscr{S}_{n}$. Thus, $\mathscr{S}$ is stable under $\alpha(A)$ and $\tau(R)$. 
(v) We define $\tau_{\mathscr{S}}$ to be the map from $R$ into the transformations of $\mathscr{S}$ given by the formula $\tau_{\mathscr{S}}(t)=\tau(t)_{\mid \mathscr{S}}$, for all $t$ in $R$. The following Proposition will be proved in Appendix 1.

Proposition 3.1. (i) $\mathscr{S}$ is a folium, $\tau_{\mathscr{S}}(R)$ is a dynamical group for $\mathscr{S}$, and $\left(\mathscr{S}, \tau_{\mathscr{S}}\right)$ is a physical folium.

(ii) If $\left(\mathscr{I}, \tau_{\mathscr{I}}\right)$ is a dynamical folium of the system, then $\left(\mathscr{I}, \tau_{\mathscr{I}}\right)$ is a physical folium if and only if it is a dynamical subfolium of $\left(\mathscr{S}, \tau_{\mathscr{S}}\right)$.

In view of this Proposition, we term $\mathscr{S}$ the set of physical states of the system and represent its dynamics by the group $\tau_{\mathscr{S}}(R)$. The physical folia of the system are simply the dynamical subfolia of $\left(\mathscr{S}, \tau_{\mathscr{S}}\right)$. Thus, the physical folia are mutually compatible, in the sense specified in Section 2.

Note. In cases where the system satisfies the HHW postulate that for all $t \in R$ and $A \in \mathscr{A}_{L}, \tau_{\Lambda}^{*}(t) A$ converges normwise as $\Lambda \rightarrow \infty$, it follows from Definition 3.2 that $\mathscr{S}=\mathscr{L}$. Thus, in these cases, the physical states are the locally normal ones.

Note. In cases where the system satisfies the DS postulates, the DS scheme is the restriction of the present one to the folia generated by the Gibbs states. For, if $\phi$ is a Gibbs state on $\mathscr{A}$, as defined in DS, then it follows easily from postulates (III) and (IV) of DS that the folium $\mathscr{I}$ generated by $\phi$ is stable under $\tau_{\mathscr{S}}(R)$. Thus, defining $\tau_{\mathscr{I}}(t) \equiv \tau_{\mathscr{S}}(t)_{\mid \mathscr{I}}$, it follows that $\left(\mathscr{I}, \tau_{\mathscr{g}}\right)$ is a physical folium. Further, it is easily shown that $\tau_{\mathscr{I}}(R)$ is precisely ${ }^{3}$ the dynamical group for the Gibbs folium $\mathscr{I}$ that is obtained from the DS postulates. Hence the present scheme is a generalisation of that of DS.

\section{Appendix 1}

Proof of Proposition 3.1. (i) It follows from Definition 3.2 (i) that $\mathscr{S}_{0}$ is a convex set; and thence by Definition 3.2 (ii), (iii) that $\mathscr{S}_{n}$ is convex for all $n \in Z_{+}$. Thus, by Definition 3.2 (iv), $\mathscr{S}$ is convex. Further, by Definition 3.2 (iii), if $\phi \in \mathscr{S}_{n}$, then $\alpha(\mathscr{A}) \phi \subset \mathscr{S}_{n-1}$ and therefore, by Definition 3.2 (iv), $\mathscr{S}$ is stable under $\alpha(\mathscr{A})$. Thus in order to prove that $\mathscr{S}$ is a folium, it suffices to establish that $\mathscr{S}$ is norm-complete. This we shall do by showing that $\mathscr{S}_{n}$ is norm-complete for all $n \in Z_{+}$.

Let $\left\{\phi_{l} \mid l \in Z_{+}\right\}$be a sequence in $\mathscr{S}_{0}$ which converges normwise to $\phi\left(\in \mathscr{A}_{+}^{*}\right)$. Then since, by Eq. (3.1), $\left\|\tau_{A}^{*}(t) A\right\| \equiv\|A\|$, it follows that $\lim _{l \rightarrow \infty}\left\langle\phi_{l} ; \tau_{A}^{*}(t) A\right\rangle=\left\langle\phi ; \tau_{\Lambda}^{*}(t) A\right\rangle \forall A \in \mathscr{A}_{A}$, the convergence being uniform

${ }^{3}$ Specifically, if $\left(\mathscr{H}_{\phi}, \pi_{\phi}, \Omega_{\phi}\right)$ is the GNS triple induced by $\phi$, then each $\psi(\in \mathscr{I})$ corresponds to a unique normal state $\tilde{\psi}$ on $\pi_{\phi}(\mathscr{A})$, with $\psi=\tilde{\psi} \circ \pi_{\phi} ;$ and $\left\langle\tau_{\mathscr{I}}(t) \psi ; A\right\rangle$ $\equiv\left\langle\tilde{\psi} ; U_{\phi}(t) \pi_{\phi}(A) U_{\phi}(-t)\right\rangle$, where $U_{\phi}(R)$ is the unitary group denoted by $\left\{\hat{U}_{t} \mid t \in R\right\}$ in DS, Proposition 3. 
with respect to $t$ and $\Lambda$. Hence, since $\left\{\phi_{l}\right\} \in \mathscr{S}_{0}$, it follows easily from Definition 3.2 (i) that $\phi \in \mathscr{S}_{0}$. Hence, $\mathscr{S}_{0}$ is norm-complete.

It also follows from the above, together with Definition 3.2 (i), that $\left\langle\tau(t)\left(\phi-\phi_{l}\right) ; A\right\rangle=\lim _{A \rightarrow \infty}\left\langle\phi-\phi_{l} ; \tau_{\Lambda}^{*}(t) A\right\rangle, \quad \forall t \in R, \quad A \in \mathscr{A}_{L}$.

Hence, since $\left\|\tau_{\Lambda}^{*}(t) A\right\| \equiv\|A\|$, it follows that

$$
\left\|\tau(t)\left(\phi-\phi_{l}\right)\right\| \leqq\left\|\phi-\phi_{l}\right\| \text {. }
$$

It is a simple consequence of this inequality, together with Definition 3.2 (ii), (iii), that $\mathscr{S}_{n}$ is norm-complete, for all $n \in Z_{+}$. Hence, by Definition 3.2(iv), $\mathscr{S}$ is norm-complete and therefore $\mathscr{S}$ is a folium. Further, by Definition 3.2(i), (iv), $\mathscr{S} \subset \mathscr{L}$; and by Definition 3.1(i)-(v), $\tau_{\mathscr{S}}$ is a homomorphism of $R$ into the affine norm-preserving transformations of $\mathscr{S}$, i.e. $\tau_{\mathscr{S}}(R)$ is a dynamical group for $\mathscr{S}$. Consequently, by Definitions $3.1,3.2(\mathrm{i}),(\mathrm{v}),\left(\mathscr{S}, \tau_{\mathscr{S}}\right)$ is a physical folium.

(ii) Suppose that $\left(\mathscr{I}, \tau_{\mathscr{I}}\right)$ is a dynamical subfolium of $\left(\mathscr{S}, \tau_{\mathscr{I}}\right)$. Then it follows directly from Definitions 3.1 and 3.2(i), (v) that $\left(\mathscr{I}, \tau_{\mathscr{I}}\right)$ is a physical folium.

Conversely, suppose that $\left(\mathscr{I}, \tau_{\mathscr{I}}\right)$ is a physical folium. Then it follows from Definitions 3.1 and $3.2\left(\right.$ i) that $\mathscr{I} \subset \mathscr{S}_{0}$ and that $\tau_{\mathscr{I}}(t)=\tau(t)_{\mid \mathscr{I}}, \forall t \in R$. Further, in view of the group property of $\tau_{\mathscr{I}}(R)$, it follows from Definition 3.2 (ii) that $\mathscr{I} \subset \mathscr{S}_{1}$, and from Definition 3.2 (iii) that $\mathscr{I} \subset \mathscr{S}_{n}, \forall n \in Z_{+}$. Hence, by Definition 3.2(iv), $\mathscr{I} \subset \mathscr{S}$. Thus we have proved that $\mathscr{I} \subset \mathscr{S}$ and that $\tau_{\mathscr{I}}(t) \equiv \tau(t)_{\mid \mathscr{I}}$, i.e. by Definition 3.2(v), that $\tau_{\mathscr{I}}(t) \equiv \tau_{\mathscr{I}}(t)_{\mid \mathscr{I}}$. In other words, $\left(\mathscr{I}, \tau_{\mathscr{I}}\right)$ is a dynamical subfolium of $\left(\mathscr{S}, \tau_{\mathscr{G}}\right)$ Q.E.D.

\section{Appendix 2}

We shall now apply the above scheme to the simple model of an ideal bose gas. Specifically, we shall show that if $\mathscr{A}$ is generated by a Weyl algebra over the Schwartz space $\mathscr{D}(\Gamma)$, then the dynamics of certain physical folia correspond to quasi-free evolutions (as defined in Ref. [10]) of a Weyl algebra over a certain subset $\tilde{\mathscr{D}}(\Gamma)(\supset \mathscr{D}(\Gamma))$ of $L^{2}(\Gamma)$. This represents a generalisation of the corresponding result obtained by DS (Appendix 1) for the islands of Gibbs states.

Let us first note that the Fock-Hilbert space $\mathscr{H}$ for an assembly of bosons may be uniquely specified, up to unitary equivalence, by the following conditions (cf. [11]).

(a) There exists a map $W$ from $L^{2}(\Gamma)$ into the unitary operators in $\mathscr{H}$ such that

$$
W(f) W(g)=W(f+g) \exp \left(\frac{1}{2} i \operatorname{Im}(f, g)\right), \quad \forall f, g \in L^{2}(\Gamma)
$$


where $(f, g)$ denotes the $L^{2}(\Gamma)$ inner product of $f$ with $g$ : thus, $W$ is a Weyl representation of $L^{2}(\Gamma)$ in $\mathscr{B}(\mathscr{H})$.

(b) There exists a vector $\Omega$ in $\mathscr{H}$ which is cyclical with respect to the $C^{*}$-algebra generated by $W\left(L^{2}(\Gamma)\right)$ and which possesses the property that

$$
(\Omega, W(f) \Omega)=\exp -\frac{1}{4}\|f\|^{2}, \quad \forall f \in L^{2}(\Gamma) .
$$

For each $\Lambda \in L$, we define $\mathscr{H}_{A}$ to be the strongly closed linear span of $W\left(L^{2}(\Lambda)\right) \Omega$, and $\mathscr{A}_{\Lambda}$ to be the $C^{*}$-algebra generated by $W(\mathscr{D}(\Lambda)$ ), with $\mathscr{D}(\Lambda)=\{f \mid f \in \mathscr{D}(\Gamma)$, supp $f \subset \Lambda\}$. It follows from these definitions that $\mathscr{A}$, the norm closure of $\bigcup_{\Lambda \in L} \mathscr{A}_{\Lambda}$, is simply the $C^{*}$-algebra generated by $W(\mathscr{D}(\Gamma))$, and that $\mathscr{A}_{A}^{\prime \prime}=\mathscr{B}\left(\mathscr{H}_{\Lambda}\right)=\left\{W\left(L^{2}(\Lambda)\right)\right\}^{\prime \prime}$.

We assume that, for each $\Lambda \in L$, the group $\tau_{A}^{*}(R)$ of automorphisms of $\mathscr{A}_{\Lambda}^{\prime \prime}$ corresponds to a quasi-free evolution of $\mathscr{A}_{\Lambda}^{\prime \prime}$, i.e., that $\tau_{\Lambda}^{\prime \prime}(R)$ is induced by a unitary representation $u_{\Lambda}$ of $R$ in $L^{2}(\Lambda)$, with

$$
\tau_{\Lambda}^{*}(t) W(f)=W\left(u_{\Lambda}(t) f\right), \quad \forall t \in R, \quad f \in L^{2}(\Lambda) .
$$

We also assume that, if $\Lambda_{0} \in L, u_{\Lambda}(t)$ converges strongly on $L^{2}\left(\Lambda_{0}\right)$, as $\Lambda \rightarrow \infty$, for each $t \in R$, i.e., that there exists a unique unitary representation $u$ of $R$ in $L^{2}(\Gamma)$, such that

$$
u(t)_{\mid L^{2}\left(\Lambda_{0}\right)}=s-\lim _{\Lambda \rightarrow \infty} u_{\Lambda}(t)_{\mid L^{2}\left(\Lambda_{0}\right)}, \quad \forall \Lambda_{0} \in L .
$$

We also assume that $u(R)$ maps $\mathscr{D}(\Gamma)$ into the Schwartz space $\mathscr{S}(\Gamma)$. These assumptions are satisfied if, for example, $u_{\Lambda}(t)=e^{i \Delta_{\Lambda} t}$ and $u(t)=e^{i \Delta t}$, where $\Delta_{A}$ is the Laplacian over $\Lambda$ with rigid or periodic boundary conditions, and where $\Delta$ is the Laplacian over $\Gamma$. In this case $u(R) \mathscr{D} \Phi \mathscr{D}$ (cf. [1; Appendix 1]).

Let $\left(\mathscr{I}, \tau_{\mathscr{I}}\right)$ be a physical folium and define $W_{\mathscr{I}}: \mathscr{D} \rightarrow \mathscr{A}_{\mathscr{I}}$ by the formula

$$
W_{\mathscr{I}}(f)=(W(f))_{\mathscr{I}}, \quad \forall f \in \mathscr{D} .
$$

We shall confine our attention to those folia possessing the following properties.

(i) If $f$ is an element of the Schwartz space $\mathscr{S}(\Gamma)$ and if $\left\{f_{n}\right\}$ is a sequence in $\mathscr{D}(\Gamma)$ which converges $(\mathscr{S})$-wise to $f$, then $\sigma\left([\mathscr{I}]^{*},[\mathscr{I}]\right)$ - $\lim _{A \rightarrow \infty} W_{\mathscr{I}}\left(f_{n}\right)$ exists and is independent of the sequence $\left\{\mathrm{f}_{n}\right\}$ in $\mathscr{D}(\Gamma)$, which is chosen to approach $f$. We define the above limit to be $W_{\mathscr{I}}(f)$, thereby extending $W_{\mathscr{I}}$ from $\mathscr{D}(\Gamma)$ to $\mathscr{S}(\Gamma)$.

(ii) If $\Lambda \in L, g \in L^{2}(\Lambda)$ and $\left\{g_{n}\right\}$ is a sequence in $\mathscr{D}(\Lambda)$ which converges $L^{2}(\Lambda)$-wise to $g$, then $\sigma\left([\mathscr{I}]^{*},[\mathscr{I}]\right)-\lim _{\Lambda \rightarrow \infty} W_{\mathscr{I}}\left(g_{n}\right)$ exists and is independent of the sequence $\left\{g_{n}\right\}$ chosen to approach $g$. We define the above limit to be $W_{\mathscr{I}}(g)$, thereby extending $W_{\mathscr{I}}$ from $\mathscr{D}(\Lambda)$ to $L^{2}(\Lambda)$. 
(iii) $\sigma\left([\mathscr{I}]^{*},[\mathscr{I}]\right)-\lim _{\Lambda \rightarrow \infty} W_{\mathscr{I}}\left(u_{\Lambda}(t) f\right)=W_{\mathscr{I}}(u(t) f), \quad \forall t \in R, \quad f \in \mathscr{D}(\Gamma)$.

(iv) $\left(\mathscr{I}, \tau_{\mathscr{I}}\right)$ is canonical, in the sense defined at the end of Section 2.

Let $\tilde{\mathscr{D}}(\Gamma)$ be the vector space of finite linear combinations of $\{u(t) f \mid t \in R, f \in \mathscr{D}(\Gamma)\}$. Then $\tilde{\mathscr{D}}(\Gamma)$ is a subset of $\mathscr{S}(\Gamma)$ and is stable under $u(R)$. Thus, it follows from Eqs. (A 2.1, 3, 4), together with Definition 3.1 and assumptions (i)-(iv), that:

(a) $W_{\mathscr{I}}(\tilde{\mathscr{D}}(\Gamma))$ is stable under $\tau_{\mathscr{\mathscr { f }}}^{*}(R)$ and that

$$
\tau_{\mathscr{I}}^{*}(t) W_{\mathscr{I}}(f)=W_{\mathscr{I}}(u(t) f), \quad \forall t \in R, \quad f \in \tilde{\mathscr{D}}(\Gamma) ;
$$

(b) $W_{\mathscr{I}}$ is a Weyl representation of $\tilde{\mathscr{D}}(\Gamma)$ in $[\mathscr{I}]^{*}$; and

(c) $\mathscr{A}_{\mathscr{I}}$ is the $C^{*}$-algebra generated by $W_{\mathscr{I}}(\mathscr{D}(\Gamma))$.

Hence, by Eqs. (A 2.1, 3, 4), time-translations in $\mathscr{I}$ correspond to the quasi-free evolution of the Weyl algebra $W_{\mathscr{I}}(\tilde{\mathscr{D}}(\Gamma))$, induced by the oneparameter transformations $u(R)$ of $\tilde{\mathscr{D}}(\Gamma)$.

\title{
References
}

1. Dubin, D. A., Sewell, G. L.: J. Math. Phys. 11, 2990 (1970)

2. Ruskai, M. B.: Commun. math. Phys. 20, 193 (1971)

3. Lanford, O.E.: Commun. math. Phys. 9, 179 (1968); 11, 257 (1967)

4. Haag, R., Hugenholtz, N. M., Winnink, M.: Commun. math. Phys. 5, 215 (1963)

5. Robinson, D.W.: Commun. math. Phys. 6, 151 (1967); 7, 337 (1968). See also Streater, R.F., Commun. math. Phys. 6, 233 (1967)

6. Haag, R., Kadison, R. V., Kastler, D.: Commun. math. Phys. 16, 81 (1970)

7. Sewell, G. L.: J. math. Phys. 11, 1869 (1970)

8. Kadison, R.V.: Topology 3, Suppl. 2, 177 (1965)

9. Fisher, M.E.: Arch. Rat. Mech. Anal. 17, 377 (1964)

10. Manuceau, J.: Ann. Inst. H. Poincaré, 8, 139 (1968)

11. Chaiken, J. M.: Commun. math. Phys. 8, 164 (1968)

\author{
G. L. Sewell \\ Department of Physics \\ Queen Mary College \\ Mile End Road \\ London E 14 NS, U.K.
}


